\title{
Wie ist die Wirksamkeit der apparativen Kompression bei Beinen mit Ödem unter Anwendung von schenkellangen und wadenlangen Stiefeln im Vergleich?
}

\section{Efficacy of intermittent pneumatic compression in the treatment of oedema comparing calf compression with calf and thigh compression devices}

\author{
Autoren \\ Erika Mendoza ${ }^{1}$, Felix Amsler ${ }^{2}$ \\ Institute \\ 1 Venenpraxis, Wunstorf \\ 2 amsler consulting, Basel, Schweiz
}

Schlüsselwörter

Intermittierenden Kompression, Entstauungsstiefel, Volumenmessungen

Key words

Intermittent pneumatic compression (IPC), leg device, volume measurements

eingereicht 28.05.2018

akzeptiert 23.01.2019

Bibliografie

DOI https://doi.org/10.1055/a-0851-0948

Phlebologie 2019; 48: 112-122

(c) Georg Thieme Verlag KG Stuttgart · New York

ISSN 0939-978X

Korrespondenzadresse

Dr. Erika Mendoza

Venenpraxis

Speckenstr. 10, Wunstorf

E-Mail: erika.mendoza@t-online.de

Englische Version unter:

https://doi.org/10.1055/a-0851-0948

\section{ZUSAMMENFASSUNG}

Einleitung Zur apparativen intermittierenden Kompression bei Ödemen aller Art sind hierzulande nur schenkellange Geräte, bzw. Hosen üblich. Wadenlange Geräte sind auf dem Markt, aber noch nicht auf ihre Wirksamkeit untersucht. Die Studie vergleicht die Wirksamkeit schenkellanger vs. knielanger Entstauungsstiefel auf Volumenreduktion und Symptome.

Methode Es handelt sich um eine forscher-initiierte, monozentrische, randomisierte Studie mit dem Vergleich der Wirksamkeit anhand Umfangmessungen der Waden (händisch und maschinell), sowie Volumenmessungen (BT 600, Bauerfeind) und Schmerz-Scores. Patienten mit Lipödem, Phlebödem, Lymphödem, Adipositas-bedingtem Ödem und medikamentös/internistischem symmetrischem Ödem wurden aufgenommen, vor der Behandlung im Stehen (nach Hinstellen, sowie nach 5 Minuten) vermessen und befragt (NRS 0-10), $30 \mathrm{Mi}-$ nuten an einem Bein (Seite randomisiert) mit langem Stiefel (Lymphamat Gradient 300, Bösl), an einer Seite mit kurzem Stiefel (Venenwalker Basic) behandelt und danach erneut vermessen und befragt.

Ergebnisse Es wurden 41 Probanden aufgenommen, davon 4 Männer, 37 Frauen, mittleres Alter 47,1 Jahre BMI 30,12 kg/m² $( \pm 5,42)$, 21 Beine „Links lang“, 20 Beine „Rechts lang“, die meisten Teilnehmer hatten ein Lipödem (26/41). Am Unterschenkel zeigten sich signifikante Verbesserungen der Umfangmaße und Volumina unabhängig von der Wahl des Stiefels, zwischen beiden Geräten waren die Unterschiede nicht signifikant. Die Wadenvolumina nahmen im Stehen zu und wurden durch die Entstauung verringert. Wir fanden eine signifikante Verringerung der Beschwerden im Stehen um 1,31 Punkte bei schenkellangem Stiefel und um 1,27 Punkte bei wadenlangem Stiefel durch die Entstauung $(p<0,001)$ ohne Differenz zwischen beiden Systemen. Es zeigte sich eine Tendenz, dass die Auffüllung des Wadenvolumens und das Verstärken der Beschwerden im Stehen nach der Entstauung langsamer geschieht.

Diskussion Üblicherweise liegen die meisten Beschwerden bei Ödemen (und auch die Komplikationen) in den Waden vor. Der wadenlange Entstauungsstiefel ist dem schenkellangen Stiefel bei der Volumen- und Beschwerdeverringerung nicht unterlegen. Möglicherweise sind bei vielen Indikationen wadenlange Entstauungsstiefel ausreichend.

\section{ABSTRACT}

Introduction In Germany intermittent pneumatic compression (IPC) to treat oedema is applied only by compressing the calf and thigh. Calf devices are available, but their efficacy was never evaluated. This investigation compared the effectivity of IPC devices only applied to the calf versus those applied to the calf and thigh. The results were expressed as leg volume change and oedema related symptoms.

Methods This was a single centre, investigator initiated, randomised study comparing the efficacy of IPC measuring calf 
and ankle perimeter electronically and by hand as well as the volume (BT 600, Bauerfeind). Pain scores with visual analogue scales from 0 to 10 were used to evaluate the symptoms. Patients with symmetric lipoedema, phleboedema, lymphoedema, oedema attributed to obesity and other medical causes were all included. Investigation consisted of measuring leg perimeters, volumes and pain scores immediately after standing as well as after 5 minutes of standing without moving. This investigation was performed before and after 30 minutes of IPC. Each patient was treated with a calf device (Venenwalker Basic) on one leg and a calf and thigh device (Lymphamat Gradient 300 , Bösl) on the other leg after the sides were randomised.

Results Forty-one patients were randomised, 4 men, 37 women, mean age 47.1 years, mean BMI $30.12 \mathrm{~kg} / \mathrm{m}^{2}( \pm 5,42), 21$ legs "left long" and 20 legs "right long”. Most of the participants had lipoedema (26/41). Both devices achieved a significant volume and perimeter reduction at calf level, no differences were found between both. Pain score were reduced significantly $(p<0.001)$ by 1.31 points after calf and thigh compression and by 1.27 points after calf compression. No significant differences were found in pain reduction between both devices. A slight tendency towards a delay in the increase of calf volume in the standing position after the treatment was shown for both devices.

Discussion Usually most discomfort and complications in case of oedema are found at the calf, where both devices showed the same volume and pain reduction. Possibly intermittent pneumatic compression applied only to the calf would be enough to reduce oedema symptoms in many cases.

\section{Einleitung}

Geräte zur apparativen intermittierenden Kompression gibt es schon seit über 100 Jahren, beschrieben 1835 von Murray und Clancy, sowie 1849 von Dr. Julius Vogel [1]. Im letzten Jahrhundert wurden sie technisch deutlich verbessert. Ihre Indikationen liegen nicht nur in der Ödemreduktion bei Lymphödem, Lipödem, Varikose sowie Ödemen anderer Ursachen [2], sondern auch in der Verbesserung der arteriellen Durchblutung bei Patienten mit arterieller Durchblutungsstörung [3, 4] und Ödemreduktion sowie Thromboseprophylaxe nach orthopädisch-unfallchirurgischen Eingriffen [5]. Um jedes Bein wird eine stiefelförmige Manschette gelegt, die sich intermittierend und nach vorgegebenen Werten von distal nach proximal mit Luft befüllt und wieder entleert, um die Flüssigkeit aus dem Bein zu streichen (S. > Abb. 1). Es gibt so genannte „Einkammer-Systeme“ und zwei unterschiedliche Systeme mit mehreren Kammern: Das eine verfügt über mehrere Generatoren, die die Kammern nacheinander befüllen, das andere über sequentielle angeordnete Kammern, über welche sich der Druck je nach Indikation vom Fuß über die Wade in den Oberschenkelbereich hinein nacheinander anfüllen, um das Ausstreichen bei der Lymphmassage zu imitieren. Bei phlebologisch-lymphologischen Indikationen sind hierzulande bisher nur Geräte mit Manschetten für Fuß-Wade-Schenkel im Einsatz (manchmal auch mit Hüft- und Bauch-Teil). Dabei führen Mehrkammer-Systeme beim Lymphödem schneller zum Erfolg, bei langfristigem Einsatz zeigt sich dann jedoch kein Unterschied zwischen Mehr- oder Einkammersystemen [6]. Die angewendeten Drucke liegen zwischen $6-124 \mathrm{mmHg}$ je nach Gerät [7], wobei für den phlebologischen Einsatz ein optimaler Druck bei 30-40 mmHg ermittelt wurde [8]. Dieser Druck wird auch bei Lymphödemen angesetzt, hier sollen maximal $60 \mathrm{mmHg}$ zum Einsatz kommen, wobei hierzu keine Studien für den optimalen Druckwert vorliegen [2] - allerdings scheinen Drucke bis zu $120 \mathrm{mmHg}$ in einigen Studien überlegen zu sein [9]. Zur Dauer der Anwendung gibt es verschiedene Studien, die meistens die AkutPhase der Entstauung betreffen und sehr variable Anwendungen von bis zu 4 und 6 Stunden pro Tag empfehlen, bzw. auch Anwendungen morgens und abends, mit verschiedenen Phasen der Kompression und Entlastung. Sie variieren zwischen 10 bis 60 Sekun- den Kompression und Pausen von 60 bis 90 Sekunden, somit ist eine Aussage zur optimalen Anwendungsweise nach Studiendaten nicht möglich [2].

Bei Einsatz im Zusammenhang mit der peripheren arteriellen Verschlusskrankheit (p-AVK) und orthopädisch postoperativen Ödemen können Fußmanschetten oder Fuß-Wadenstiefel eingesetzt werden [2-5].

Klassische Indikationen für die apparative intermittierende Entstauung innerhalb der Phlebologie sind:

- Lipödem: unbekannte Ursache, möglicherweise familiär mit Dysproportion zwischen Rumpf und Beinen (10).

- Lymphödem: Ödem durch Verzögerung des Lymphabflusses, typischerweise so genannten „Kastenzehen“ (Stemmerzeichen), das Ödem hat eine eher teigige Konsistenz.

- Phlebödem: Durch Krampfadern bedingte Schwellung des Beines

- Adipositas-bedingtes Ödem (AbÖ): Lymphödem durch Verzögerung des Abflusses in der Leiste und Retroperitonäum [11-13].

- Weitere Ursachen für Ödeme: Viele Medikamente und Krankheiten können Ödeme verursachen, jedoch auch das lange unbewegte Stehen oder Sitzen, sowie eine eingeschränkte Beweglichkeit der Muskulatur [9].

Die Therapie aller Ödeme ist, soweit möglich, die Ausschaltung der Ursache, sowie die Kompression (als Bandagierung) und später als Kompressionsstrümpfe, kombiniert mit manueller Lymphdrainage oder apparativer Entstauung, je nach Ausprägungsgrad. Nach der ersten Entstauungsphase mit Bandagierung und häufigen Lymphmassagen (komplexe physikalische Entstauung) wird eine langanhaltende Therapiephase zum Erhalt des Ergebnisses eingeleitet mit Kompressionsstrümpfen und je nach Bedarf Lymphdrainage oder Entstauung, wenn die Ursache für das Ödem nicht therapierbar ist.

Klassischerweise wurde bei Kompressionsstrümpfen immer die schenkellange Variante gewählt, um Varizen, Thrombosen und auch Ödeme zu therapieren, bis festgestellt wurde, dass die knielangen Kompressionsstrümpfe zum Vorbeugen der Hautver- 
änderungen bei Patienten venöser Insuffizienz (z. B. Varikose, Z. n. Thrombose) ausreichend wirksam sind [15].

Die apparative intermittierende Kompressionstherapie (AIK) bei Lymphödem wird üblicherweise mit schenkellangen Manschetten mit bis zu 12 Kammern oder mit Entstauungshosen durchgeführt. Die zunehmend auftretenden Wadenödeme bei Lipödem oder adipositasbedingtem Ödem werden seit Jahren auch mit wadenlangen Geräten behandelt, die frei käuflich sind. Ihre Effektivität bei unfallchirurgischen Patienten wurde bereits belegt [4]. Ein direkter Vergleich der Wirksamkeit beider Gerätetypen auf das chronische Ödem und die Lebensqualität bzw. die Symptome ist bisher jedoch nicht erfolgt. Die wadenlangen Manschetten sind in der Anwendung einfacher für den Patienten, platzsparend und in der Anschaffung deutlich kostengünstiger.

\section{Ziel der Studie}

Vergleich der Wirkung von schenkellangen und wadenlangen Entstauungsstiefeln auf das Wadenvolumen und das subjektive Wohlbefinden, bzw. die Symptomlinderung des Patienten.

\section{Methode}

Es handelt sich um eine Forscher-initiierte, monozentrische, randomisierte Studie zum Bestätigen oder Widerlegen der Hypothese: „Schenkellange und wadenlange Entstauungsgeräte haben eine ähnliche Wirksamkeit auf das Wadenödem und seine Symptome“. Die primäre Zielgröße ist der Vergleich der Umfangmaße bzw. des Volumens der Waden nach Anwendung des schenkellangen oder wadenlangen Entstauungsgerätes gemessen mit dem Bandmaß an Knöchel und Wade, sowie mit dem BT 600 (Firma Bauerfeind). Die sekundäre Zielgrößen sind der Vergleich der mittels einer Analogskala gemessenen Symptomänderung und der mittels eines Fragebogens erfasste Bedienungskomfort der Geräte. Die Ethik-Kommission der Ärztekammer Niedersachsen sah keinen Beratungsbedarf für die Durchführung der Studie. Die Patienten erhielten eine mündliche Aufklärung, ein schriftliches Informationsblatt, sowie eine schriftliche Einverständniserklärung.

\section{Probanden}

Die Probanden wurden aus den Patienten der Praxis rekrutiert, entweder durch direkte Ansprache oder durch ein Anschreiben nach Sichtung der Unterlagen der Patientenakte. Einschlusskriterien waren das Vorliegen eines symmetrischen Ödems folgender Ursachen: Lymphödem Grad I-II, Lipödem Grad I-II, Phlebödem, Adipositas-bedingtes Ödem analog Grad I-II des Lymphödems und weitere symmetrische Ödeme internistischer Ursachen.

Ausschlusskriterien waren eine regelmäßige Therapie mit Lymphmassage, Alter unter 18 oder über 80, Schwangerschaft, schwere internistische Erkrankung, orthopädische Probleme, die ein schnelles Aufstehen oder längeres Stehen erschweren, Teilnahme an anderen Studien, Schwierigkeiten in der Verständigung auf Deutsch (Fragebögen) sowie Nicht-Einwilligung .

\section{Datenerfassung}

Alle Untersuchungen erfolgten zwischen 14 und 18 Uhr. Die Patienten wurden gebeten, am Untersuchungstag, und möglichst ein paar Tage davor, keine Kompressionsstrümpfe zu tragen. Am Untersuchungstag wurden folgende Schritte durchgeführt:

1. Diagnose und der Ultraschallbefund der Beinvenen (Reflux in der tiefen oder oberflächlichen Beinvene, ggf. Hach-Stadium), sowie das Alter aus der Patientenakte erfasst und das Gewicht sowie die Körpergröße erhoben.

2. Patientenfragebogen zur Ausprägung der Beschwerden durch die Schwellung, Dauer der Schwellung, Tragen von Kompression und Art der Kompression.

3. Abfragen folgender Symptome der vergangenen Woche getrennt nach rechtem und linken Bein: Druckgefühl, Spannungsgefühl, Schmerzgefühl, Krämpfe, Juckreiz, Kribbeln, Ziehen, Brennen, Schweregefühl, Schwellung, Stechen, Hitzegefühl oder Müdigkeit in den Beinen, bzw. weitere Symptome, die angegeben werden konnten.

4. Scoring der Beschwerden insgesamt in der letzten Woche je Bein und für Wade und Oberschenkel getrennt. Dafür wurde eine 5-stufige Likert Skala von „Überhaupt keine“ bis „sehr stark" verwendet.

5. Nun wurde der Proband gebeten, sich hinzulegen und die Beine auf einem $50 \mathrm{~cm}$ hohen Schaumstoffkissen für 2 Minuten hoch zu lagern.

6. Stellen auf den Bodytronic 600 (Firma Bauerfeind) zum elektronischen Vermessen der Beine. Dort sollte er circa 7 Minuten unbewegt stehen.

7. Sofort nach dem Hinstellen und erneut nach 5-6 Minuten wurden elektronisch die Volumen der Unter- und der Oberschenkel gemessen.

8. Während der je eine Minute dauernden ersten und zweiten Messung wurden die Patienten zu ihren Beinschmerzen, getrennt nach rechts und links sowie Oberschenkel und Wade, mit einer numerischen Analogskala (NRS) von 0 bis $10 \mathrm{ge}$ fragt („NRS Stehen vorher“ 1 und 2, daraus wurde der Mittelwert „Stehen vorher MW“ gebildet).

9. Nach der ersten elektronischen Messung erfolgte eine händische Messung des Knöchel- und des oberen Wadenumfangs (B-Maß, D-Maß).

10. Randomisierung in die Gruppe „rechts lang“ oder „links lang“, mit der festgelegt wurde, an welchem Bein die schenkellange bzw. die wadenlange Manschette angelegt wird.

11. Zum Anlegen der Entstauungsstiefel legte sich der Proband auf eine bequeme Liege. An einem Bein wurde entsprechend der Randomisierung der lange Stiefel, an dem anderen der kurze Stiefel angelegt.

12. Der Patient wurde mit einem Fragebogen nach dem Befinden im Liegen gefragt, getrennt nach linkem und rechten Bein an Wade und Oberschenkel („NRS Liegen vorher“). Er durfte während der 30 Minuten der Behandlung nicht aufstehen und bei Bedarf ein Glas (200 ml) Wasser trinken, jedoch keine anderen Getränke, insbesondere keine harntreibenden. Er durfte lesen, Musik hören oder anderweitig entspannen. Hätte er während der 30 Minuten aufstehen müssen, wäre die Studie für diesen Tag abgebrochen und bei Wunsch des Patienten 

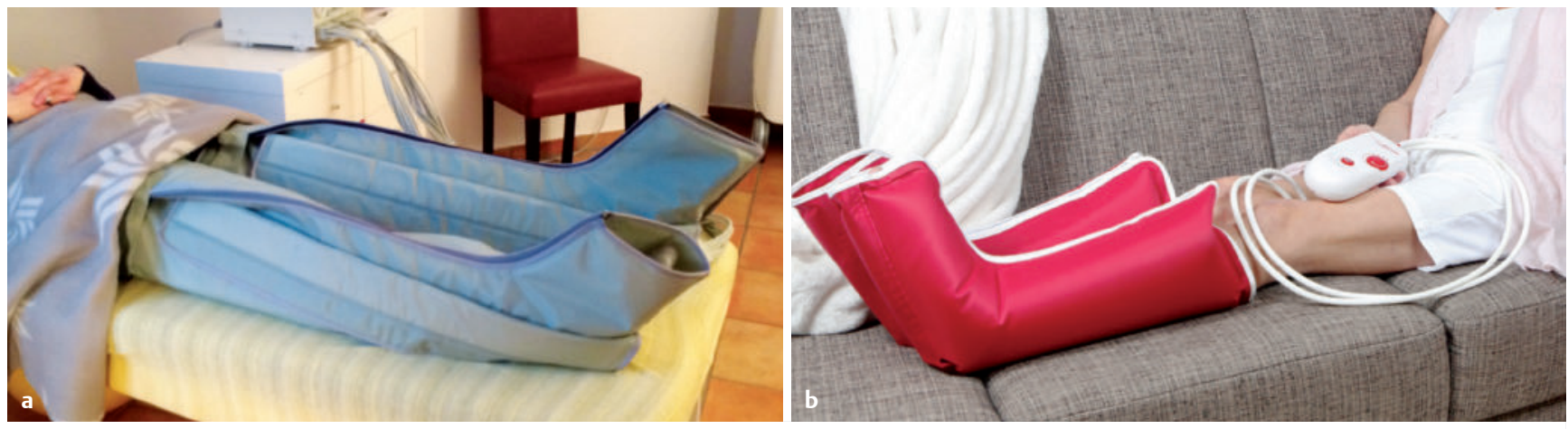

- Abb. 1 Fotos der für die Studie verwendeten Manschetten. a Schenkellange 6-Kammer-Manschette (Lymphamat Gradient 300 der Firma Bösl); b Wadenlange Manschette der Firma Globalmind, Venenwalker Basic (Quelle: Globalmind consumer electronics GmbH).

- Tab. 1 Demographische Daten der 41 Studien-Patienten.

\begin{tabular}{|l|l|l|l|l|l|}
\hline & Mittel & Median & $\begin{array}{l}\text { Standard- } \\
\text { Abweichung }\end{array}$ & $\begin{array}{l}\text { Mini- } \\
\text { mum }\end{array}$ & $\begin{array}{l}\text { Maxi- } \\
\text { mum }\end{array}$ \\
\hline $\begin{array}{l}\text { Alter } \\
\text { (Jahre) }\end{array}$ & 47,10 & 48 & 12,64 & 21 & 75 \\
\hline $\begin{array}{l}\text { Größe } \\
\text { (cm) }\end{array}$ & 167,63 & 167 & 5,28 & 160 & 187 \\
\hline $\begin{array}{l}\text { Gewicht } \\
\text { (kg) }\end{array}$ & 84,53 & 83 & 15,49 & 52 & 120 \\
\hline $\begin{array}{l}\text { BMl } \\
\text { (kg/cm }\end{array}$ & 30,12 & 30,85 & 5,42 & 17,4 & 40,7 \\
\hline
\end{tabular}

an einem anderen Tag erneut durchgeführt worden (dies ist nicht eingetreten).

13. Kurz vor Ende der Entstauung wurde der Patient zum zweiten Mal nach dem Befinden im Liegen gefragt (NRS Liegen während).

14. Nach Ende der Entstauungsphase wiederholte sich die Messreihe wie vor der Entstauung (elektronisch, manuell, elektronisch, sowie Fragen zu Anfang und Ende des 5-minütigen Stehens: „NRS Stehen vorher“ 1 und 2, daraus der Mittelwert "Stehen vorher MW“).

15. Abschließende Fragen, ob einer der beiden Stiefel wirksamer erschien und ob sie einen der beiden Stiefel empfehlen würden.

\section{Geräte}

Bei dem langen Stiefel handelt es sich um ein 6-Kammer System „Lymphamat Gradient 300“ der Firma Bösl ( $>$ Abb. 1b), eingestellt auf einen Druck von $40 \mathrm{mmHg}$. Bei dem kurzen Stiefel handelt es sich den Venenwalker basic ( $\triangleright$ Abb. 1a), ein Zweikammersystem (Fußsohlenkammer und Wadenkammer) der Firma Global-Mind, geeicht auf $40 \mathrm{mmHg}$.

\section{Statistik}

Es gab zur Berechnung einer Power-Analyse keine Vergleichsdaten. Aufgrund der Wirkung von Kompressionsstrümpfen auf Bein-
- Tab. 2 Verteilung der Ödem-Typen bei der Studienpopulation.

\begin{tabular}{|l|l|l|}
\hline Ödem-Typ & Häufigkeit & Prozent \\
\hline Lipödem & 26 & 63,4 \\
\hline Lymphödem & 5 & 12,2 \\
\hline Phlebödem & 1 & 2,4 \\
\hline Lip- \&Lymphödem & 2 & 4,9 \\
\hline Lip-\&Phlebödem & 2 & 4,9 \\
\hline Lymph-\&Phlebödem & 3 & 7,3 \\
\hline Medikamentös oder internistisch & 2 & 4,9 \\
\hline Gesamt & $\mathbf{4 1}$ & $\mathbf{1 0 0 , 0}$ \\
\hline
\end{tabular}

volumina konnte geschätzt werden, dass eine Auswertung nach 40-60 Probanden möglich sein würde, daher wurden 40-60 Probanden geplant, mit der Option, die Untersuchung nach 40 Probanden abzuschließen

Die Studie wurde als zweifach gepaarte Vergleichsstudie durchgeführt, indem die Werte vor der Behandlung mit denjenigen nach der Behandlung und die Werte des Beines mit dem kurzen Stiefel mit denjenigen des langen Stiefels verglichen wurden. Dazu wurde die Wirkung der Behandlung als Differenz zwischen den Messungen vor und nach der Entstauung operationalisiert. Die Resultate werden als Mittelwerte und Standardabweichung dargestellt. Für die NRS-Skalen und die Umfänge und Volumen wurden T-Tests für gepaarte Stichproben mit zweiseitigen Signifikanztests wurden durchgeführt, für die übrigen Einschätzungen Wilcoxon-Rangtests: $p<0,05$ wurde als statistisch signifikant gewertet. Die Auswertung erfolgte mit SPSSTM for Windows 24 (Armonk, NY: IBM Corp, USA).

\section{Ergebnisse}

Es nahmen 41 Probanden an der Studie teil, davon 4 Männer und 37 Frauen. Das mittlere Alter lag bei 47,1 Jahren der mittlere BMI bei $30,1 \mathrm{~kg} / \mathrm{m}^{2}$ ( Tab. 1). Im Mittel hatten die Patienten seit 12,1 Jahren geschwollene Beine ( $\pm 12,5$, Min 1, Max 56, Median 8 Jahre). $95 \%$ der Patienten trugen regelmäßig Kompressionsstrümpfe, davon $56 \%$ wadenlange. 21 Beine wurden in die Gruppe „Links lang“ randomisiert, 20 Beine in die Gruppe „Rechts lang“. 4 Pro- 
- Tab. 3 Beschwerden in der letzten Woche vor Behandlung in einer visuellen Analog Skala von 0 bis 10, sowie Vergleich der Beschwerden zwischen der Gruppe „Lang“ und „kurz“ (p-Wert in der rechten Säule) (MW = Mittelwert, SD = Standard-Abweichung, $\mathrm{p}=$ Signifikanz).

\begin{tabular}{|l|l|l|l|}
\hline Stiefeltyp & lang & kurz & \\
\hline Beschwerden & $\mathbf{M W} \pm \mathbf{S D}$ & $\mathbf{M W} \pm \mathbf{S D}$ & $\mathbf{P}$ \\
\hline Schweregefühl & $3,08 \pm 0,97$ & $2,98 \pm 1,00$ & 0,378 \\
\hline Schwellung & $2,88 \pm 1,01$ & $2,83 \pm 0,92$ & 0,570 \\
\hline Müdigkeit & $2,70 \pm 1,22$ & $2,68 \pm 1,27$ & 0,661 \\
\hline Spannungsgefühl & $2,48 \pm 0,82$ & $2,48 \pm 0,88$ & 1,000 \\
\hline Druckgefühl & $2,33 \pm 1,07$ & $2,25 \pm 1,06$ & 0,446 \\
\hline Schmerzgefühl & $2,18 \pm 1,04$ & $2,20 \pm 1,22$ & 0,785 \\
\hline Hitzegefühl & $1,81 \pm 1,24$ & $1,84 \pm 1,34$ & 0,711 \\
\hline Kribbeln & $1,80 \pm 0,94$ & $1,75 \pm 0,93$ & 0,623 \\
\hline Brennen & $1,69 \pm 1,06$ & $1,67 \pm 1,08$ & 0,767 \\
\hline Ziehen & $1,56 \pm 0,91$ & $1,62 \pm 0,94$ & 0,421 \\
\hline Krämpfe & $1,54 \pm 0,72$ & $1,51 \pm 0,68$ & 0,767 \\
\hline Juckreiz & $1,45 \pm 0,65$ & $1,58 \pm 0,89$ & 0,058 \\
\hline Stechen & $1,38 \pm 0,91$ & $1,41 \pm 0,91$ & 0,711 \\
\hline Beschwerden Wade & $\mathbf{2 , 6 5 \pm 1 , 1 9}$ & $\mathbf{2 , 5 3} \pm \mathbf{1 , 1 5}$ & $\mathbf{0 , 1 3 3}$ \\
\hline Beschwerden OS & $\mathbf{1 , 9 0 \pm 0 , 9 6}$ & $\mathbf{1 , 9 0} \pm \mathbf{0 , 8 4}$ & $\mathbf{1 , 0 0 0}$ \\
\hline & & & \\
\hline
\end{tabular}

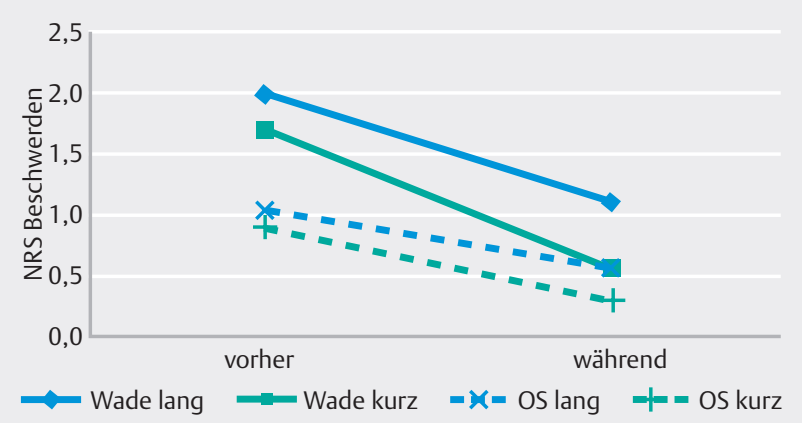

Abb. 2 Beschwerden im Liegen vor der Behandlung und während der Behandlung (Vgl. > Tab.4) Alle Veränderungen sind im Vergleich vorher zu nachher signifikant, die Gruppen unterscheiden sich nicht voneinander. NRS = Werte der visuellen Analog-Scala von 0 bis 10 für Numeric Rating Scale. Wade Lang: Gruppe mit langem Stiefel, gemessen an der Wade, Wade kurz: Gruppe mit kurzem Stiefel, gemessen an der Wade. OS Lang: Gruppe mit dem langen Stiefel, gemessen am Oberschenkel, OS Kurz: Gruppe mit dem kurzen Stiefel, gemessen am Oberschenkel.

banden hatten am Tag der Untersuchung bereits an beiden Beinen Kompressionsstrümpfe getragen.

Die Aufteilung der Ödem-Typen ist in Tabelle 2 wiedergegeben.

Ein Phlebödem lag bei 5 Beinen mit langem Stiefel und 4 Beinen mit kurzem Stiefel vor, alle zeigten ein $C(C E A P)$ von $C_{3}$, bis auf ein Bein in der Gruppe „Lang“ mit C4a. In beiden Gruppen waren 2

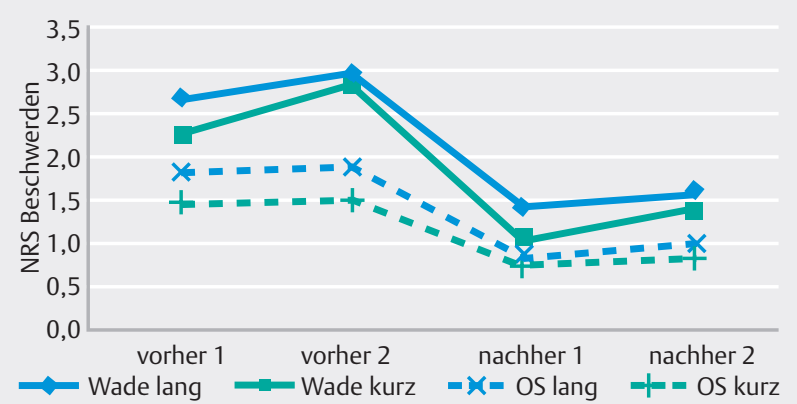

Abb. 3 Entwicklung der Beschwerden im Stehen unmittelbar nach dem Hinstellen (vorher 1 und nachher 1 ) und nach 5 Minuten unbewegtem Stehen (vorher 2 und nachher 2 ) vor und nach der Behandlung (vgl. \ Tab.4). Alle Veränderungen sind im Vergleich vorher zu nachher signifikant, die Gruppen unterscheiden sich nicht voneinander. NRS $=$ Werte der visuellen Analog-Scala von 0 bis 10. Wade Lang: Gruppe mit langem Stiefel, gemessen an der Wade, Wade kurz: Gruppe mit kurzem Stiefel, gemessen an der Wade. OS Lang: Gruppe mit dem langen Stiefel, gemessen am Oberschenkel, OS Kurz: Gruppe mit dem kurzen Stiefel, gemessen am Oberschenkel.

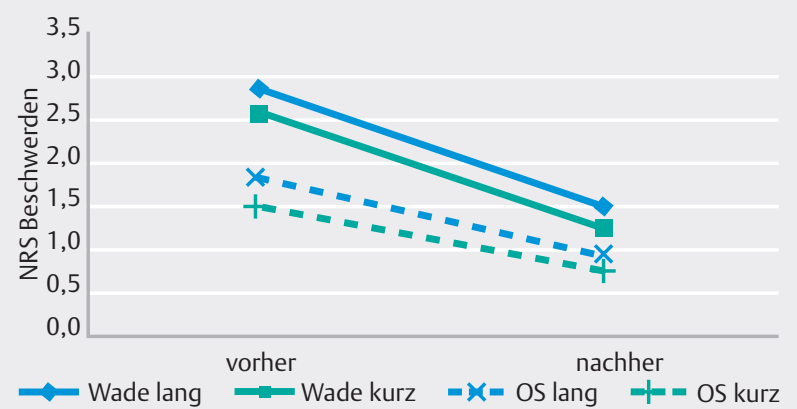

- Abb.4 Beschwerden im Stehen (MW der beiden Messungen) vor und nach der Behandlung (Vgl. — Tab.4). Alle Veränderungen sind im Vergleich vorher zu nachher signifikant, die Gruppen unterscheiden sich nicht voneinander. NRS = Werte der visuellen Analog-Scala von 0 bis 10. Wade Lang: Gruppe mit langem Stiefel, gemessen an der Wade, Wade kurz: Gruppe mit kurzem Stiefel, gemessen an der Wade. OS Lang: Gruppe mit dem langen Stiefel, gemessen am Oberschenkel, OS Kurz: Gruppe mit dem kurzen Stiefel, gemessen am Oberschenkel.

Beine voroperiert (Z. n. Stripping der V. saphena magna), ein Bein in der Gruppe „Lang“ hatte einen Reflux in der tiefen Beinvene.

Die Beschwerden in der letzten Woche vor der Behandlung sind in $>$ Tab. 3 dargestellt. Das Symptom mit der stärksten Ausprägung war das Schweregefühl, gefolgt von Schwellung und Müdigkeit der Beine. Die Einstufung der Beschwerden insgesamt lag bei circa 2,5 Punkten für die Waden und 1,9 für die Oberschenkel. Die Beine in beiden Gruppen unterschieden sich nicht in Bezug auf die Beschwerden. Die Entwicklung der Beschwerden vor, während und nach der Untersuchung ist in $>$ Tab. 4, sowie $>$ Abb. 2- Abb. 4 dargestellt.

Die Beschwerden im Liegen ( $>$ Abb. 2, $>$ Tab.4) an der Wade verringerten sich zwischen vorher und während signifikant für 
- Tab. 4 Entwicklung der Beschwerden auf der visuellen Analog Scala (NRS 0-10). Erfasst werden die Daten im Liegen vor und während der Behandlung, sowie die Werte im Stehen vor und nach der Behandlung mit Mittelwert und Standardabweichung je Bein, unterteilt in Säulen nach Beinen mit langem Stiefel und kurzen Stiefel. In der 3. Säule wird der Unterschied zwischen beiden Beinen, bzw. dem kurzen und dem langen System erfasst und in der 4. Säule die Signifikanz dieser Veränderung. Es wird das Ergebnis des kurzen Stiefels vom langen Stiefel abgezogen, somit zeigen positive Werte bei der Abweichung der Mittelwerte bessere Ergebnisse zugunsten des kurzen Stiefels, negative Werte zeigen bessere Ergebnisse zugunsten langem Stiefel. In den Zeilen wird der absolute Wert je Bein angegeben, 1.Zeile vor der Behandlung, zweite Zeile während oder nach der Behandlung, sowie in der 3. Zeile die mittlere Differenz des Wertes im selben Bein für die Untersuchung im Liegen vor und während der Behandlung sowie im Stehen vor und nach der Behandlung. Gezeigt werden beide Messungen (Vorher/während bzw. vorher/Nachher) mit Mittelwert (MW) und Standardabweichung (SD)). In der jeweils 3. Zeile wird die Differenz vor und während, bzw. nach der Behandlung je Bein ausgerechnet. In allen Fällen gab es eine Verringerung der Werte, sodass das Ergebnis mit einem „minus“ Zeichen gekennzeichnet sind. Die 4. Zeile zeigt die Signifikanz der Differenz. * Signifikante Ergebnisse.

\begin{tabular}{|c|c|c|c|c|}
\hline & \multirow{2}{*}{$\begin{array}{l}\text { lang } \\
\mathrm{MW} \pm \mathrm{SD}\end{array}$} & \multirow{2}{*}{$\begin{array}{l}\text { kurZ } \\
M W \pm S D\end{array}$} & \multicolumn{2}{|l|}{ lang - kurz } \\
\hline & & & MW Diff. \pm SD & $\mathbf{p}$ \\
\hline \multicolumn{5}{|l|}{ Wade } \\
\hline NRS Liegen vorher & $1,99 \pm 2,3$ & $1,72 \pm 2,08$ & $0,27 \pm 1,29$ & 0,192 \\
\hline NRS Liegen während & $1,12 \pm 2,05$ & $0,57 \pm 1,25$ & $0,55 \pm 1,31$ & $0,011^{*}$ \\
\hline Mittlere Diff. vorher während & $-0,87 \pm 2,36$ & $-1,15 \pm 1,87$ & $0,28 \pm 1,91$ & 0,352 \\
\hline P vorher/während & $0,024^{*}$ & $<0,001^{*}$ & & \\
\hline NRS Stehen vorher 1 & $2,68 \pm 2,58$ & $2,28 \pm 2,26$ & $0,40 \pm 1,29$ & 0,057 \\
\hline NRS Stehen nachher 1 & $1,44 \pm 1,82$ & $1,05 \pm 1,6$ & $0,39 \pm 1,27$ & 0,062 \\
\hline Mittlere Diff. vorher nachher & $-1,31 \pm 1,67$ & $-1,27 \pm 1,67$ & $-0,04 \pm 1,05$ & 0,821 \\
\hline p vorher/nachher & $<0,001^{*}$ & $<0,001^{*}$ & & \\
\hline NRS Stehen vorher 2 & $3,00 \pm 2,56$ & $2,83 \pm 2,26$ & $0,17 \pm 1,43$ & 0,448 \\
\hline NRS Stehen nachher 2 & $1,58 \pm 2,08$ & $1,42 \pm 1,84$ & $0,15 \pm 1,23$ & 0,438 \\
\hline Mittlere Diff. vorher nachher & $-1,21 \pm 2,04$ & $-1,24 \pm 1,96$ & $0,04 \pm 1,08$ & 0,825 \\
\hline p vorher/nachher & $0,001^{*}$ & $<0,001^{*}$ & & \\
\hline NRS Stehen vorher MW & $2,86 \pm 2,49$ & $2,59 \pm 2,19$ & $0,27 \pm 1,27$ & 0,173 \\
\hline NRS Stehen nachher MW & $1,53 \pm 1,79$ & $1,25 \pm 1,50$ & $0,28 \pm 1,17$ & 0,132 \\
\hline Mittlere Diff. vorher nachher & $-1,33 \pm 1,79$ & $-1,34 \pm 1,75$ & $0,01 \pm 0,92$ & 0,966 \\
\hline p vorher/nachher & $<0,001^{*}$ & $<0,001^{*}$ & & \\
\hline \multicolumn{5}{|l|}{ Oberschenkel } \\
\hline NRS Liegen vorher & $1,05 \pm 1,78$ & $0,89 \pm 1,45$ & $0,16 \pm 1,24$ & 0,426 \\
\hline NRS Liegen während & $0,61 \pm 1,14$ & $0,29 \pm 0,78$ & $0,32 \pm 0,85$ & $0,022^{*}$ \\
\hline Mittlere Diff. vorher während & $-0,44 \pm 1,66$ & $-0,60 \pm 1,2$ & $0,16 \pm 1,44$ & 0,478 \\
\hline p vorher/während & 0,100 & $0,003^{*}$ & & \\
\hline NRS Stehen vorher 1 & $1,84 \pm 2,65$ & $1,46 \pm 2,12$ & $0,38 \pm 1,27$ & 0,070 \\
\hline NRS Stehen nachher 1 & $0,85 \pm 1,27$ & $0,75 \pm 1,24$ & $0,10 \pm 1,17$ & 0,593 \\
\hline Mittlere Diff. vorher nachher & $-1,01 \pm 2,04$ & $-0,73 \pm 1,82$ & $-0,28 \pm 1,41$ & 0,220 \\
\hline P vorher/nachher & $0,004^{*}$ & $0,016^{*}$ & & \\
\hline NRS Stehen vorher 2 & $1,89 \pm 2,63$ & $1,52 \pm 2,14$ & $0,37 \pm 1,24$ & 0,066 \\
\hline NRS Stehen nachher 2 & $1,04 \pm 1,68$ & $0,82 \pm 1,43$ & $0,22 \pm 1,52$ & 0,375 \\
\hline Mittlere Diff. vorher nachher & $-0,69 \pm 1,9$ & $-0,60 \pm 1,77$ & $-0,09 \pm 1,81$ & 0,759 \\
\hline p vorher/nachher & $0,028^{*}$ & $0,040^{*}$ & & \\
\hline NRS Stehen vorher MW & $1,84 \pm 2,61$ & $1,48 \pm 2,10$ & $0,37 \pm 1,22$ & 0,063 \\
\hline NRS Stehen nachher MW & $0,93 \pm 1,35$ & $0,76 \pm 1,26$ & $0,18 \pm 1,23$ & 0,362 \\
\hline Mittlere Diff. vorher nachher & $-0,91 \pm 2,03$ & $-0,72 \pm 1,83$ & $-0,19 \pm 1,47$ & 0,416 \\
\hline p vorher/nachher & $0,007^{*}$ & $0,016^{*}$ & & \\
\hline
\end{tabular}

beide Stiefel, die Beschwerden am Oberschenkel verringerten sich nur für den langen Stiefel signifikant. Während der Behandlung waren die Beschwerden mit dem kurzen Stiefel sowohl an der Wade als auch am Oberschenkel signifikant niedriger als mit dem langen $(p<0,05)$. Insgesamt unterschied sich die mittlere Differenz, also die Wirksamkeit der Behandlung der beiden Stiefel jedoch nicht signifikant.

Die Beschwerden im Stehen ( $\triangleright$ Abb. $\mathbf{3}$ und $\triangleright$ Abb. 4, $\triangleright$ Tab. 4) verbesserten sich nach der Behandlung signifikant im Vergleich zu den Messungen vor der Behandlung, bei Messung 1 (sofort nach 

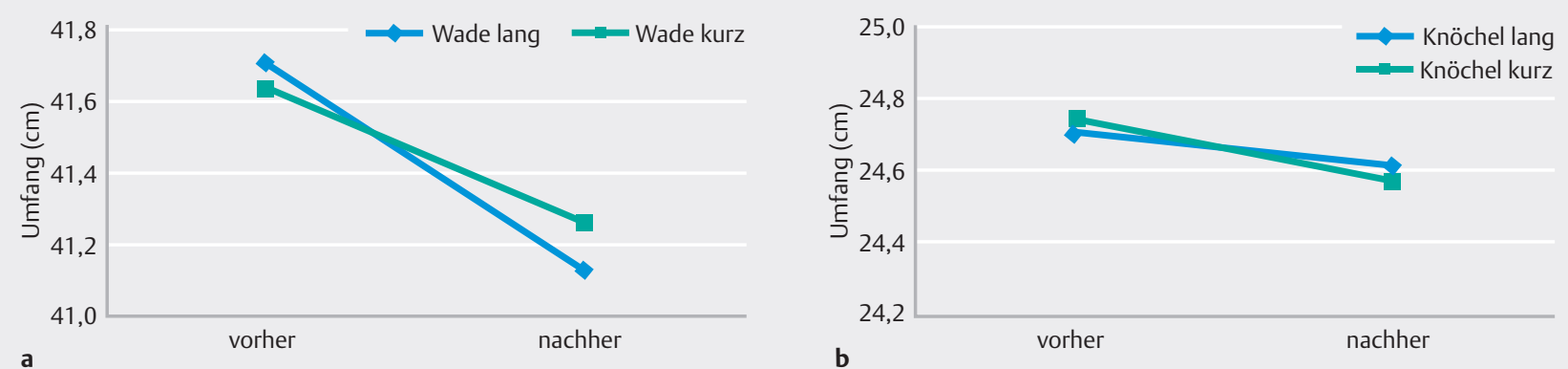

> Abb. 5. Veränderung des Umfangs an der Wade (a) und am Knöchel (b) vor und nach der Behandlung (Vgl. \ Tab.4). Mit Ausnahme des Knöchelmaßes an den Beinen mit langem Stiefel sind alle Änderungen vorher zu nachher signifikant, es liegen zwischen den Gruppen „Kurz“ oder „Lang“ keine Unterschiede vor. Wade Lang: Gruppe mit langem Stiefel, gemessen an der Wade, Wade kurz: Gruppe mit kurzem Stiefel, gemessen an der Wade. Knöchel Lang: Gruppe mit dem langen Stiefel, gemessen am Knöchel, Knöchel Kurz: Gruppe mit dem kurzen Stiefel, gemessen am Knöchel.

\Tab. 5 Entwicklung des Umfangs an Wade und Knöchel $(\mathrm{cm})$ vor und nach der Behandlung. Erklärung zu den Feldern siehe $>$ Tab. 4. * Signifikante Ergebnisse.

\begin{tabular}{|l|l|l|l|l|}
\hline & lang & kurz & lang - kurz \\
\hline & MW \pm SD & MW \pm SD & MW Diff, \pm SD \\
\hline Umfang Wade vorher & $41,71 \pm 4,10$ & $41,64 \pm 4,12$ & $0,07 \pm 0,91$ & 0,608 \\
\hline Umfang Wade nachher & $41,13 \pm 4,17$ & $41,26 \pm 4,20$ & $-0,13 \pm 1,19$ & 0,482 \\
\hline Mittlere Diff, \pm SD & $-0,58 \pm 0,86$ & $-0,38 \pm 0,97$ & $\mathbf{- 0 , 2 0 \pm 0 , 9 9}$ & $\mathbf{0 , 1 9 2}$ \\
\hline P vorher/nachher & $<0,001^{*}$ & $0,017^{*}$ & & 0,722 \\
\hline Umfang Knöchel vorher & $24,71 \pm 1,99$ & $24,75 \pm 2,07$ & $-0,04 \pm 0,65$ \\
\hline Umfang Knöchel nachher & $24,62 \pm 1,87$ & $24,58 \pm 2,00$ & $0,04 \pm 0,48$ & 0,586 \\
\hline Mittlere Diff, \pm SD & $-0,10 \pm 0,38$ & $-0,17 \pm 0,38$ & $\mathbf{0 , 0 8 \pm 0 , 5 3}$ & $\mathbf{0 , 3 4 9}$ \\
\hline P vorher/nachher & 0,121 & $0,006^{*}$ & & \\
\hline
\end{tabular}

dem Hinstellen), 2 (nach 5 Minuten) und dem MW, sowohl an der Wade $(p=0,001$ oder $<0,001)$ als auch in geringerem Ausmaß am Oberschenkel ( $p$ zwischen 0,004 und 0,04). Die beiden Systeme unterschieden sich nicht signifikant voneinander, weder am Unterschenkel noch am Oberschenkel.

Die Umfangmaße vor und nach der Behandlung sind in \ Tab. $\mathbf{5}$ und $\ \mathbf{A b b} \mathbf{5}$ dargestellt. Mit Ausnahme des Umfangs des Knöchels unter dem langen Stiefel waren alle gemessenen Veränderungen in der erwarteten Richtung signifikant. Die Unterschiede der Ergebnisse zwischen den beiden Stiefel-Modellen waren nicht signifikant.

Die Wadenvolumina ( $>$ Tab. 6, $\triangleright$ Abb. 6) nahmen im Stehen zu und wurden durch die Entstauung verringert. Der Mittelwert der beiden Messungen verringerte sich bei beiden Stiefeln signifikant nach der Behandlung. Es zeigte sich eine Tendenz, dass die Auffüllung des Wadenvolumens nach der Entstauung langsamer geschieht. Die Oberschenkelvolumina nahmen beim ersten Stehen für beide Gruppen zu, nach der Entstauung mit dem langen Gerät nahm das Volumen am langen Stiefel signifikant ab, am kurzen blieb es praktisch konstant, der Unterschied war nicht signifikant. Beide Oberschenkelvolumen nahmen nach der Behandlung im Lauf der 5 Minuten unbewegten Stehens ab ( $\triangleright$ Abb. 6).
Zur Beurteilung, ob ein Unterschied zwischen den beiden Geräten vorliegt (Unterlegenheit/Nicht-Unterlegenheit) wurden die Differenzen der Mittelwerte in Bezug auf Beschwerdeskala, Umfang und Volumen errechnet. A Abb. 7 zeigt graphisch die Mittelwerte und die $95 \%$-Vertrauensintervalle der Differenzen. Alle Unterschiede sind weit weg von einer Signifikanz, das heißt, das 95\%-Intervall liegt nie gesamthaft auf einer Seite der Null-Linie.

In der persönlichen Einschätzung nach Abschluss der Behandlung gaben die Probanden an, dass sie den langen Stiefel als signifikant effektiver empfanden als den kurzen $(p<0,001)$, den kurzen jedoch eher als angenehmer ( $p=0,225)$. In Bezug auf die Wade fiel diese Unterscheidung nicht signifikant aus mit folgenden Aussagen: Für 17 Teilnehmer war der lange Stiefel an der Wade effektiver, für 8 der kurze. Als gleichwertig in Bezug auf die Wirkung an der Wade entschieden sich 15 Teilnehmer, 1 empfand keinen als effektiv. Eine Patientin, welche in die Auswertung nicht einbezogen wurde, brach die Studie wegen Schmerzen unter dem langen Stiefel ab.

Die Frage nach der Weiterempfehlung, ebenfalls am Ende des zweiten Steh-Versuchs gestellt, ergab 14 positive Empfehlungen für den schenkellangen Stiefel, 15 Empfehlungen für den wadenlangen, 10 für beide gleich und 1 für keinen ohne Signifikanz zugunsten des einen oder anderen Modells. 

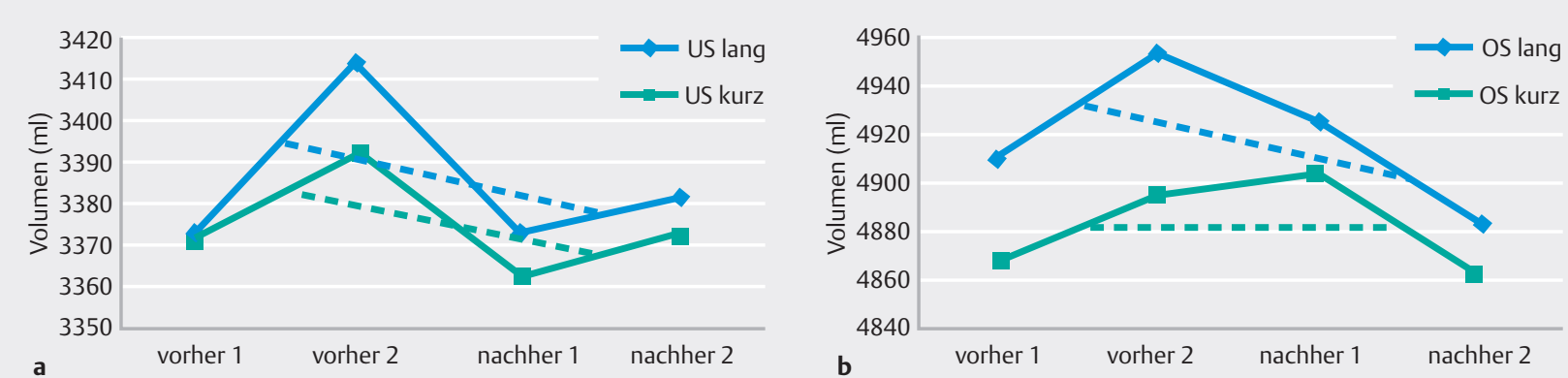

- Abb. 6 Veränderung des Waden- und des Oberschenkelvolumens, gemessen mit dem BT 600, jeweils zweimalig vor und nach der Behandlung sowie Mittelwert der beiden Messungen (gepunktete Linie) (vgl. > Tab. 6). Signifikante Volumenreduktion an der Wade beidseits, am Oberschenkel nur in der Gruppe „Lang“. Wade Lang: Gruppe mit langem Stiefel, gemessen an der Wade, Wade kurz: Gruppe mit kurzem Stiefel, gemessen an der Wade. OS Lang: Gruppe mit dem langen Stiefel, gemessen am Oberschenkel, OS Kurz: Gruppe mit dem kurzen Stiefel, gemessen am Oberschenkel.

- Tab. 6 Entwicklung des Volumens ( $\mathrm{ml}$ ) am Unterschenkel und am Oberschenkel (Mittelwert der beiden Messungen) vor und nach der Behandlung. Erklärungen s. $\triangleright$ Tab. 4. * Signifikante Ergebnisse.

\begin{tabular}{|l|l|l|l|l|}
\hline & lang & kurz & lang - kurz \\
\hline Variable & MW \pm SD & MW \pm SD & MW Diff. \pm SD \\
\hline Vol US vorher & $3394 \pm 625$ & $3383 \pm 627$ & $11 \pm 103$ & 0,514 \\
\hline Vol US nachher & $3378 \pm 620$ & $3368 \pm 618$ & $10 \pm 113$ & 0,592 \\
\hline Mittlere Diff. \pm SD & $-16 \pm 50$ & $-15 \pm 46$ & $-1 \pm 45$ & $\mathbf{0 , 8 7 7}$ \\
\hline P vorher/nachher & $0,047^{*}$ & $0,049^{*}$ & $51 \pm 248$ & 0,197 \\
\hline Vol OS vorher & $4932 \pm 967$ & $4882 \pm 982$ & $21 \pm 230$ & 0,562 \\
\hline Vol OS nachher & $4904 \pm 945$ & $4883 \pm 976$ & $-\mathbf{3 0} \pm \mathbf{1 4 3}$ & $\mathbf{0 , 1 8 9}$ \\
\hline Mittlere Diff. \pm SD & $-28 \pm 89$ & $2 \pm 155$ & \\
\hline P vorher/nachher & $0,048^{*}$ & 0,948 & \\
\hline
\end{tabular}

\section{Diskussion}

\section{Homogenität der Gruppen}

Die Diagnosen und Symptome an den Beinen die Woche vor der Behandlung der beiden Gruppen („lang“ und „kurz“) unterschieden sich nicht ( Tab. 3 und $\triangleright$ Tab. 4). Da wir einen Vergleich zwischen zwei Beinen bei derselben Person vorgenommen haben, liegen natürlich in Bezug auf Alter und Geschlecht keine Unterschiede vor, mit 20 zu 21 Probanden „lang rechts“ vs. „lang links“ ist die Randomisierung ebenfalls homogen verlaufen.

\section{Größe der Stichprobe}

Die Auswertung nach Aufnahme von 41 Probanden ergab ausreichend hohe Signifikanzen, sodass die Größe als ausreichend gewertet werden konnte für die Beantwortung der vorhandenen Fragestellung.

\section{Wertigkeit der gemessenen Parameter}

Die Erhebung der Beschwerdezunahme und der Wadenvolumina im Stehen basiert auf dem Stehversuch mit dem BT 600 mit gesunden Probanden von Blättler et al. [16]. Dort lag die mittlere Zunahme des Wadenvolumens nach 10 Minuten Stehen bei 44 ml, der Kom- pressionsstrumpf Klasse II verringerte diese Zunahme um die Hälfte $(22 \mathrm{ml})$. Die Beschwerdezunahme im Stehen ohne Kompression lag bei 2,9 Punkten im Mittelwert, die Zunahme mit Kompression bei 2,3 Punkten.

Die Beschwerdezunahme in dieser Studie wurde gemessen zwischen Anfang und Ende des Stehens (nur 5 Minuten statt 10 wie bei Blättler) sowohl vor, als auch nach der Behandlung. Außerdem wurde der Mittelwert beider Messungen (Anfang und Ende des Stehversuchs vor, sowie Anfang und Ende des Stehversuchs nach der Behandlung) verglichen ( $>$ Tab.4).

Der Mittelwert der Wadenbeschwerden verringerte sich um 1,33 (Lang) respektive 1,34 Punkte (kurz) durch die Behandlung, beides $p<0,001$ und beides ohne Unterschied zwischen den verschiedenen Stiefeln. Betrachtet man die Beschwerdezunahme in den ersten Minuten nach dem Hinstellen (s. $>$ Abb. 6), nimmt diese vor der Behandlung mit 0,32 (Wade lang) und 0,55 (Wade kurz) nicht signifikant zu, wie bei dem Stehversuch von Blättler, jedoch zeigen dort die Graphiken, dass die Beschwerdezunahme relativ linear ist und daher in dieser Studie eine geringere Wirkung zu erwarten ist, da statt 10 nur 5 Minuten erhoben wurden. Interessant scheint, dass das Beschwerdebild zwischen vor und nach der Behandlung deutlich abnimmt, dass also beide Geräte eine Verringe- 

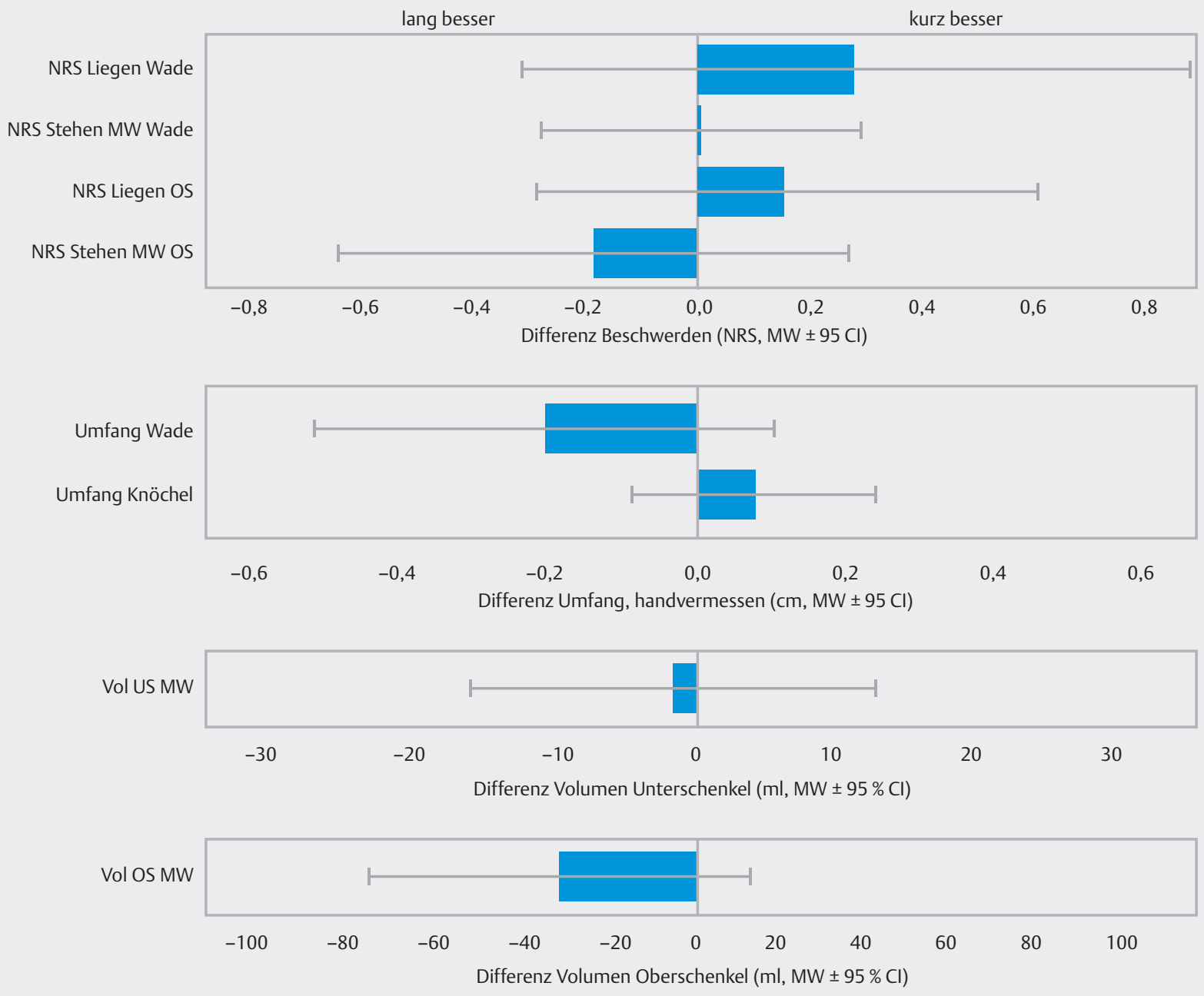

- Abb.7 Mittelwert und 95\% Vertrauensintervall der Unterschiede vor und nach der Behandlung (Stehen) bzw. vor und während der Behandlung (Liegen) zwischen dem langen und dem kurzen Stiefel. NRS = Werte der visuellen Analog-Scala von 0 bis 10. OS= Oberschenkel, US= Unterschenkel, $\mathrm{MW}=$ Mittelwert, $\mathrm{Vol}=$ Volumen .

rung der Wadenbeschwerden unmittelbar nach Hinstellen um 1.31 (lang) und 1,27 (kurz) Punkte hervorrufen, sowie ebenso nach $5 \mathrm{Mi}$ nuten Stehens (je 1,21 und 1,24 Punkte), beides hoch signifikant. Auch zeigt sich nach der Behandlung eine langsamere Beschwerde-sowie Volumenzunahme in der Wade ( $\vee$ Abb. 3 und $\triangleright$ Abb. 6). Die vorliegenden Studienergebnisse korrelieren mit den Ergebnissen aus der Blättler-Studie[16], somit kann angenommen werden, dass sie zunächst kohärent zu den Erwartungen sind und kein Systemfehler vorliegt.

\section{Tragweite der Fragestellung}

Mit einer einmaligen Behandlung kann die Nachhaltigkeit der Wirkung auf das Alltagsleben nicht festgestellt werden. Dies war aber auch nicht Sinn dieser Studie. Ähnlich aber wie bei anderen therapeutischen Eingriffen in der Medizin im Allgemeinen (z. B. Tabletten verabreichen), als auch in der Therapie des Ödems im Konkreten (Bandagieren, Kompressionsstrumpf tragen, Lymphmassage) kann gesagt werden, dass die Wirkung dieser Maßnahmen nur so lange vorhält, wie das Medikament wirkt oder die Anwendung vor- hält, bzw. möglicherweise Stunden oder Tage danach (bei Kompression und Lymphmassage).

Daher wurde hier zunächst die Effektivität des Wadenstiefels im Vergleich zum schenkellangen Stiefel als einmaliger Eingriff erfasst. Es ist jedoch davon auszugehen, dass die Wirksamkeit an wiederholten Tagen ebenso stark ist, wie das Tragen von Kompressionsstrümpfen oder Wiederholen von schenkellangen Entstauungsanwendungen über mehrere Tage.

Die Zielgruppe hatte ein nicht ausgeprägtes Ödem, da es ethisch bedenklich gewesen wäre, Patienten mit schwerem Ödem das Tragen von Kompression für einen Tag vorzuenthalten. Es ist jedoch davon auszugehen, dass analog zur Wirkung des Kniestrumpfs bei schweren Ödemen auch die Waden-Entstauung eine Wirksamkeit bei schwereren Ödemen haben wird. Diese Hypothese müsste allerdings in einer separaten Studie noch geprüft werden.

\section{Kurioses Ergebnis}

Überraschend, wenngleich auch nachvollziehbar, ist die Entwicklung der Volumina in den Oberschenkeln (s. $>$ Abb. 6). Beim ersten Stehversuch nehmen sie erwartungsgemäß zu. Nach Entstau- 
ung mit der schenkellangen Manschette nehmen sie ab, mit der wadenlangen Manschette zu - verständlich, das Volumen aus der Wade wurde nur in das nächste Kompartiment verlagert, nämlich bei dem schenkellangen Gerät aus dem Oberschenkel in das Becken und somit verringert, und bei dem wadenlangen Gerät aus der Wade in den Oberschenkel, wodurch das Volumen im Oberschenkel erhöht wurde.

Spannend ist die 4. Messung - nach dem Stehen nach der Behandlung und unabhängig von der Art der Manschette, fällt das Oberschenkelvolumen ab. Eine mögliche Erklärung wäre denkbar: Der Oberschenkel füllt sich sowohl venös als auch lymphatisch aus der Wade. Nach Entstauung der Wade wäre dieses nachfließende Volumen reduziert. Entleert sich der Oberschenkel normal weiter nach proximal (venös und lymphatisch), ohne einen Nachschub aus der Wade, wird das Oberschenkelvolumen zunächst abnehmen. Dies Phänomen tritt für beide Gruppen in ähnlicher Weise auf (die Linien in \ Abb. 6 Volumen OS zwischen „Nachher 1 “ und „Nachher 2“ verlaufen parallel für beide Geräte).

Überraschend ist, dass dieses Volumen in beiden Fällen auf einen niedrigeren Wert als dem Ausgangswert fällt. Somit kann für beide Geräte gesagt werden, dass auch Volumen aus dem Oberschenkel aus dem Bein verlagert wurde.

Vergleicht man das mittlere Volumen des gesamten Beins zu Anfang der Untersuchung („Vorher 1“: lang 8.284 ml, kurz 8.240 ml) mit dem am Ende (nach dem Stehen nach der Behandlung, „Nachher 2“: lang $8.266 \mathrm{ml}$, kurz $8.236 \mathrm{ml}$ ), sieht man, dass beide diskret niedriger sind, beim langen Stiefel um $18 \mathrm{ml}$, beim kurzen um nur $4 \mathrm{ml}$ - die Unterschiede sind nicht signifikant und sehr klein im Vergleich zur Streuung zwischen beiden Geräten. Somit kann wohl auch für den wadenlangen Stiefel eine Wirkung auf das gesamte Bein erzielt werden, das über das Verlagern des Volumens aus der Wade in den Oberschenkel hinaus geht.

\section{Anwendbarkeit in der Klinik}

Es wurden für diese Studie Probanden mit einem nicht ausgeprägten Ödem verschiedener Ursachen gewählt. Diese Zielgruppe nimmt in unserer Bevölkerung zu, aufgrund der zunehmenden Adipositas [11-13] und aufgrund der vielen Stunden, in denen wir in der so genannten zivilisierten Welt stehen oder sitzen und somit unsere Muskelpumpe nicht aktivieren. Zusätzlich zu dem objektivierbaren Ödem liegen Symptome vor, die möglicherweise unabhängig von der Ödemzunahme die Lebensqualität beeinträchtigen, wie sehr eindrücklich von Blazek et al. an einem Kollektiv an Friseuren ohne venöse Insuffizienz gezeigt werden konnte. Kompression verbesserte signifikant die Lebensqualität, und zwar unabhängig von der Tatsache, dass sie auch einen Einfluss auf das Wadenvolumen hatte [17].

Eine Symptomverringerung bei unbewegtem Stehen konnte nach Anwendung der wadenlangen Entstauungsstiefel analog zu den schenkellangen nachgewiesen werden. Es liegt also nahe, dass die kurzen Entstauungsstiefel für die Symptome von Menschen, die diese Beschwerden nur an der Wade verspüren, ausreichend sind. Die Volumenverringerung erfolgte an der Wade ohne Unterschiede in beiden Gruppen - daher kann der wadenlange Stiefel bei Patienten mit Ödemen ausschließlich an der Wade eingesetzt werden und wird hier keinen Nachteil zu schenkellangen Geräten haben.
Eine Untersuchung bei höheren Schweregraden des Ödems wäre weiterführend interessant.

Bei Patienten mit Ganzbeinödemen sind die Beschwerden an der Wade aufgrund der Orthostase in der Regel ausgeprägter. Die häufigste Versorgung für diese Patienten in Deutschland ist allenfalls die Kompressionsstrumpfversorgung. Selten wird eine komplexe physikalische Entstauungstherapie durchgeführt, noch seltener langfristig eine Lymphmassage rezeptiert, da dies von den Kassenärztlichen Vereinigungen mit Budgetüberschreitungen und drohenden Regressen für die Ärzte geahndet wird. Bis diese Unterversorgung systematisch gelöst werden kann, wird der Patient aber davon profitieren, ein Gerät ggf. selbst erwerben zu können, dessen Anschaffungskosten deutlich geringer sind als die sonst handelsüblichen.

Somit kann als Vorteil für die Bevölkerung (sprich, für die Umsetzung in der alltäglichen Praxis) genannt werden, dass sowohl bei Dyskomfort durch Zivilisationsprobleme (Sitzen, Adipositas), Beschwerden in den Waden als auch Ödemen in den Waden die Entstauung mit finanziell zugänglichen wadenlangen Entstauungsgeräten - optimalerweise in Kombination mit Kompressionsstrümpfen - besser ist als keine Therapie, insbesondere da sie in Bezug auf die Symptome der Wade den schenkellangen Geräten gleichwertig ist. Die erste Aussage (besser als nichts) trifft möglicherweise auch bei Ganzbeinödemen zu.

Der Nachweis der Volumenreduktion und Verringerung der Beschwerden mit einem kleinen wadenlangen Gerät in diesem nicht sehr symptomatischen Kollektiv legt nahe, dass eine große Bevölkerungsgruppe von dieser kleinen Lösung schon ausreichend profitieren würde.

Der Nachweis wäre nun noch in Patientenkollektiven zu führen, deren Symptome ausgeprägter sind.

\section{ZUSAMMENFASSUNG}

Sowohl die gemessenen Beschwerden durch Beinschwellung als auch die Beinumfangmaße und Beinvolumina verringern sich durch die Behandlung mit Entstauungsstiefeln signifikant. Dabei konnten keine signifikanten Unterschiede zwischen wadenlangen und schenkellangen Geräten festgestellt werden, weder in Bezug auf die Waden noch in Bezug auf die Oberschenkel.

\section{Danksagung}

Ich danke der Firma Bauerfeind, dass ich den BT 600 für die Studie verwenden durfte. Ich danke Dr. Igor Zolotukin (Univ. Moskau) für den konstruktiven Input für die Erklärung der Volumenverringerung am Oberschenkel im Stehen nach der Entstauung.

Interessenkonflikt

Die statistische Auswertung durch FA wurde von der Firma GlobalMind bezahlt, EM hat keine Interessenkonflikte. 


\section{Literatur}

[1] Vogel J. Die Hämospasie, Giessen 1852

[2] Schwahn-Schreiber C, Reich-Schupke S, Breu FX et al. S1-Leitlinie Intermittierende Pneumatische Kompression (IPK,AIK). Hautarzt 2018; 69: 662-673

[3] Delis KT, Nicolaides N. Effect of intermittent pneumatic compression of foot and calf on wal- king distance, hemodynamics and quality of life in patients with arterial claudication. Ann Surg 2005; 241: 431-41

[4] Labropoulos N, Wierks C, Suffoletto B. Intermittent pneumatic compression for the treatment of low extremity arterial disease: a systematic view. Vasc Med 2002; 7: 141-8

[5] Tamir L, Hendel D, Neyman C et al. Sequential foot compression reduces low limb swelling and pain after total knee arthroplasty. | Arthroplasty 1999; 14: 333-8

[6] Bergan J], Sparks S, Angle N. A comparison of compression pumps in the treatment of lymphedema. J Vasc Surg 1998; 32: 455-62

[7] Rithalia SVS, Heath GH, Gonsalkorale M. Evaluation of intermittent pneumatic compression systems. J Tissue Viability 2002; 12: 52-7

[8] Grieveson S. Intermittent pneumatic compression pump settings for the optimum reduction of oedema. J Tissue Viability 2003; 13: 98-110

[9] Taradaj J, Rosińczuk J, Dymarek R et al. Comparison of efficacy of the intermittent pneumatic compression with a high- and low-pressure application in reducing the lower limbs phlebolymphedema. Therapeutics and Clinical Risk Management 2015; 11: 1545-1554
[10] Reich-Schupke S, Schmeller W, Brauer W] et al. Leitlinie Lipödem; J. Dtsch Dermatol Ges 2017; 15 (7): 758-768. doi: 10.1111/ ddg.13036_g

[11] Göstl K, Obermayer A, Hirschl M, Pathogenese der chronisch venösen Insuffizienz durch Adipositas - Aktuelle Datenlage und Hypothesen, Phlebologie 2009; 38: 108-113

[12] Färber G, Adipositas und chronische Inflammation bei Phlebologischen und Lymphologischen Erkrankungen. Phlebologie 2018; 47: 55-65 https://doi.org/10.12687/phleb2413-2-2018

[13] Bertsch T, Adipositas assoziierte Lymphödeme - unterschätzt und unterbehandelt, Phlebologie 2018; 47: 75-83 https://doi.org/10.12687/ phleb2410-2-2018

[14] Mendoza E, Duplex Ultrasound in the Diagnosis of Leg Swelling, Reviews in Vascular Medicine 2015; 3: 17-23

[15] Prandoni P, Noventa F, Quintavalla R et al. Thigh-length versus below-knee compression elastic stockings for prevention of the postthrombotic syndrome in patients with proximal-venous thrombosis: a randomized trial; Blood 2012; 119 (6): 1561-5

[16] Blättler W, Thomä HJ, Winkler C et al. Leichte Medizinische Kompressionsstrümpfe reduzieren Beschwerden beim Stehen gleich gut wie straffe; Phlebologie 2016; 45: 25-28

[17] Blazek C, Amsler F, Blaettler W et al. Compression hosiery for occupational leg symptoms and leg volume: a randomized crossover trial in a cohort of hairdressers; Phlebology 2013; 28 (5): 239-47. doi: 10.1258/phleb.2011.011108 